\title{
Um estudo sobre os modelos de grandes deformações em corpos elásticos
}

\author{
Covre, L. ${ }^{1^{*}} ;$ Loeffler, C. F. ${ }^{2}$ \\ 1 Departamento de Engenharia Mecânica, Universidade Federal do Espírito Santo, Vitória, ES, Brasil \\ 2 Programa de Pós-Graduação em Engenharia Mecânica, Universidade Federal do Espírito Santo, Vitória, ES, Brasil. \\ *e-mail: lucas_loriato@hotmail.com
}

\begin{abstract}
Resumo
Este trabalho examina e compara os modelos de deformação não linear de Green e a deformação verdadeira (True Strain). Em algumas situações não tão incomuns, a hipótese de pequenas deformações em modelos de engenharia estrutural não pode ser aplicada. Isto ocorre quando ocorre significativa mudança na conformação geométrica do corpo, relativamente à configuração original. Para pequenas deformações, ambos os modelos são aproximadamente iguais, mas para grandes deformações, o comportamento é completamente diferente: enquanto o modelo de Green prediz menores deslocamentos em relação ao modelo linear, a deformação verdadeira prevê maiores deslocamentos. Aqui são analisadas as particularidades dos dois modelos não-lineares e demonstra-se que o modelo de Green para os casos uniaxiais é matematicamente inconsistente, enquanto que o modelo de deformação verdadeira apresenta uma série de predicados, entre os quais: a possibilidade de considerar as deformações plásticas inerentes ao processo; a capacidade de inclusão no modelo da variação da área transversal do corpo; a sua consistência associativa, quando o carregamento total é aplicado em etapas distintas.
\end{abstract}

\begin{abstract}
This work examines and compares the models of nonlinear Green Strain and True Strain. In some nonunusual situations, the hypothesis of small strains in structural engineering models cannot be applied. This occurs when there is a significant change in the geometric shape of the body relatively to the original shape configuration. For small deformations, both models are approximately equal, but for large deformations, the behavior is completely different: while the Green model predicts smaller displacements with respect to the linear model, the true strain predicts greater displacements. Here the particularities of the two nonlinear models are examined and it is demonstrated that the Green model for one dimensional cases is mathematically inconsistent, whereas the true strain model presents a series of predicates, among which: the possibility of considering the inherent plastic deformations along the process; the capability for inclusion of the cross-sectional area variation of the body in the model; the associative consistency when the loading is applied in distinct steps.
\end{abstract}

Keywords: Large Strain; Green Model for Strain; True Strain.

\section{Introdução}

A teoria das grandes deformações é essencial para a mecânica dos sólidos, pois fornece os recursos matemáticos necessários para descrever o comportamento mecânico de elementos tais como cordas, cabos, chapas e corpos em geral sujeitos à deformação plástica. Estes sólidos sofrem alterações significativas em sua conformação geométrica e estas não são validadas pela teoria das deformações infinitesimais, em que se assume que não ocorrer alteração significativa entre as configurações original e deformada do corpo.

\section{Descrição Matemática Deformação}

\subsection{Modelo Geral}

Uma sequência de configurações que resulta do movimento de um corpo flexível pode ser descrita a partir de uma configuração de referência $X$, onde a nova configuração $x$ é dada por um operador $K$ de modo que [1]:

$$
\boldsymbol{x}=K\left(\boldsymbol{X}_{r}, \mathrm{t}\right)
$$


Este operador K está sujeito, entre outras condições, a seguinte restrição [1]:

$$
\mathrm{I}=\operatorname{det} \mathrm{K}>0
$$

Como as diferentes configurações são, em realidade, transformações no estado do corpo, e o determinante de uma transformação dá a ideia da conservação ou não do volume, a condição $\mathrm{J}>0$ exclui da categoria de deformação qualquer movimento que desintegre 0 corpo. Assim:

$$
\begin{aligned}
& 0<\mathrm{J}<1 \rightarrow \text { Contrações } \\
\mathrm{J}= & \rightarrow \quad \text { Movimentos de corpo rígido } \\
\mathrm{J}>1 & \rightarrow \text { Expansões }
\end{aligned}
$$

Define-se como deformação o mapeamento de uma configuração sobre outra configuração. Para melhor apreensão, admita duas configurações distintas de um determinado corpo $\Omega(X)$. No interior deste corpo, considere dois pontos distanciados de um infinitésimo dX, conforme apresentado na Figura 1:

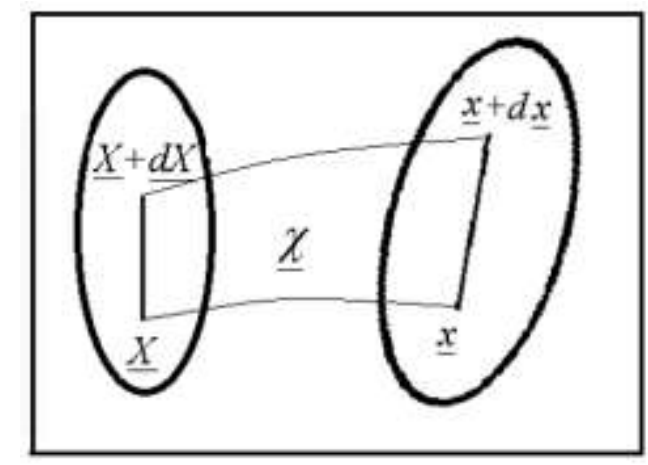

Figura 1: Mudança da configuração inicial de um determinado corpo.

As relações entre os pontos na configuração inicial e deformada podem ser expressas pelo operador $\mathrm{K}$. Então:

$$
\begin{aligned}
\mathrm{x} & =\mathrm{K}(\mathrm{X}, \mathrm{t}) \\
\mathrm{x}+\mathrm{dx} & =\mathrm{K}(\mathrm{X}+\mathrm{dX}, \mathrm{t})
\end{aligned}
$$

Pela definição do gradiente:

$$
\mathrm{K}(\mathrm{X}+\mathrm{dX}, \mathrm{t})-\mathrm{K}(\mathrm{X}, \mathrm{t})=\nabla \mathrm{K}(\mathrm{X}, \mathrm{t}) \mathrm{dX}
$$

Logo:

$$
\mathrm{dx}=\nabla \mathrm{K}(\mathrm{X}, \mathrm{t}) \mathrm{dX}=\mathrm{FdX}
$$

Assim, o diádico definido por $F(X, t)$, chamado de gradiente de deformação, transforma um elemento $d X$ na configuração de referência em um elemento $d x$ na configuração deformada [2].

\subsection{O Campo de Deslocamentos}

Denomina-se campo de deslocamentos o campo vetorial $u(X, t)$ definido pela relação:

$$
\mathrm{u}(\mathrm{X}, \mathrm{t})=\mathrm{x}-\mathrm{X}=\mathrm{K}(\mathrm{X})-\mathrm{X}
$$

É claro que o movimento do corpo de uma região para outra no espaço pode preservar as distâncias ou o mapeamento relativo entre os pontos. Seria este o movimento de corpo rígido. Contudo, se nessa alteração de posição no espaço as distancias relativas se alterarem, há deformação no sentido usual. Assim, aplicando-se o operador gradiente na expressão dos deslocamentos, obtém-se:

$$
\nabla \mathrm{u}(\mathrm{X}, \mathrm{t})=\nabla \mathrm{K}(\mathrm{X}, \mathrm{t})-\nabla \mathrm{X}=\mathrm{F}-\mathrm{I}
$$

Pode-se depreender por esta última expressão que numa transformação de uma configuração em outra que o gradiente de $u(X, t)$ representa a alteração relativa às configurações.

\subsection{Medidas de Deformação}

$\mathrm{Na}$ busca de uma medida para quantificar-se a deformação, que é uma grandeza diádica, é interessante efetuar o seguinte produto escalar:

$$
\mathrm{dx} \cdot \mathrm{dx}=(\mathrm{FdX}) \cdot(\mathrm{FdX})=\mathrm{F}^{\mathrm{T}} \mathrm{FdX} \cdot \mathrm{dX}=\mathrm{CdX} \mathrm{dX}
$$

Considerando que:

$$
\begin{aligned}
& d x \cdot d x=d s^{2} \\
& d x \cdot d x=d s^{2}
\end{aligned}
$$

Pode-se estabelecer a relação entre comprimentos antes e depois do movimento:

$$
d s^{2}-d S^{2}=(C-I) d X, d X=2 E d X, d X
$$

Na equação (12), E é o denominado Tensor de Green [2], que fornece uma medida conveniente, embora arbitrária, para se expressar a deformação. Além disso, sua consistência é atestada pelo seu uso ostensivo em muitas aplicações na engenharia.

Ressalta-se que não se pode obter uma diferença de comprimentos no sentido de uma norma euclidiana, que é a medida mais usual na representação da alteração no valor de qualquer grandeza escalar, como os comprimentos antes e depois da deformação. No caso, isto se deve a aplicação de um tratamento tridimensional geral numa grandeza tensorial de segunda ordem, como a deformação. 
Uma forma bastante estratégica de se apresentar o Tensor de Green consiste da seguinte expressão [3,4]:

$$
\mathrm{E}=\frac{1}{2}\left(\overline{V u}+\nabla \mathbf{u}^{\mathrm{T}}+\nabla \mathbf{u} \nabla \mathrm{u}^{\mathrm{T}}\right)
$$

Na engenharia estrutural, é muito comum considerar-se pequenas diferenças entre as configurações deformada e original. A escolha de materiais rígidos é fundamental para assegurar a validade desta hipótese. Assim, extremamente aplicado na Mecânica dos Sólidos é o chamado tensor de pequenas deformações $\varepsilon$, um tensor simétrico, que é dado por [5]:

$$
\varepsilon=\frac{1}{2}\left(\nabla u+\nabla u^{T}\right)
$$

Mais comum na Mecânica dos Fluidos, mas também importante na Mecânica dos Sólidos, é o tensor das rotações $\omega$, um tensor antissimétrico [2]:

$$
\omega=\frac{1}{2}\left(\overline{\nabla u}-\nabla u^{T}\right)
$$

O tensor de Green E pode ser escrito estrategicamente em termos dos tensores supracitados:

$$
E=\varepsilon+\frac{1}{2}\left(\varepsilon^{2}-\omega^{2}+\varepsilon \omega-\omega \varepsilon\right)
$$

\subsection{Casos Particulares}

É comum encontrar-se as seguintes simplificações:

(a) Pequenos alongamentos e grandes rotações:

$$
\mathrm{E}=\varepsilon+\frac{1}{2}\left(\varepsilon \omega-\omega \varepsilon-\omega^{2}\right)
$$

(b) Pequenos alongamentos com rotações moderadas:

$$
\mathrm{E}=\varepsilon-\frac{1}{2}\left(\omega^{2}\right)
$$

(c) Pequenos alongamentos e pequenas rotações:

$$
\mathrm{E}=\varepsilon
$$

(d)Grandes alongamentos e pequenas rotações:

$$
E=\varepsilon+\frac{1}{2}\left(\varepsilon^{2}\right)
$$

E é exatamente sobre esta última expressão que se fará uma análise mais apurada.

\section{Análise do Tensor de Green}

No caso unidimensional em que se aplicam movimentos axiais, não há rotação, e assim a Eq. (9) se particulariza fornecendo:

$$
\begin{array}{r}
\mathrm{dx} \cdot \mathrm{dx}=\mathrm{ds^{2 }}=\left(1+\frac{\partial \mathrm{u}}{\partial \mathrm{x}_{1}}\right)^{2} \mathrm{dx}_{1}^{2} \\
=\left(1+\frac{\partial \mathrm{u}}{\partial \mathrm{x}_{1}}\right)^{2} \mathrm{dS}^{2}=\left[1+\frac{2 \partial \mathrm{u}}{\partial \mathrm{x}_{1}}+\left(\frac{\partial \mathrm{u}}{\partial \mathrm{x}_{1}}\right)^{2}\right] \mathrm{dS}^{2}
\end{array}
$$

Tal como mostra a Eq. (19), faz-se:

$$
\mathrm{E}_{\mathrm{x} 1}=\left(\frac{\partial \mathrm{u}}{\partial \mathrm{x}_{1}}\right)+\frac{1}{2}\left(\frac{\partial \mathrm{u}}{\partial \mathrm{x}_{1}}\right)^{2}
$$

Então:

$$
d x \cdot d x=d s^{2}=\left[1+2 E_{x I}\right] d s^{2}
$$

Contudo, sendo o problema unidimensional é possível usar a norma euclidiana e calcular ds diretamente:

$$
\mathrm{d} s=\left(1+\frac{\partial \mathrm{u}}{\partial \mathrm{x}_{1}}\right) \mathrm{dx}_{1}=\left(1+\frac{\partial \mathrm{u}}{\partial \mathrm{x}_{1}}\right) \mathrm{ds}
$$

Então:

$$
d s-d S=\frac{\partial u}{\partial x_{1}} d x_{1}=\frac{\partial u}{\partial x_{1}} d S_{1}=\varepsilon_{x 1} d S_{1}
$$

A conclusão é evidente: o tensor $E$ no caso de grandes alongamentos inclui o quadrado do tensor de pequenas deformações $\varepsilon$ de modo artificial, pois no caso geral não seria viável obter uma expressão euclidiana para a medida da deformação.

\section{A Deformação Verdadeira}

\subsection{Modelo Básico}

Em problemas unidimensionais, uma interessante medida de deformação se baseia na atualização do valor do comprimento da peça quando sujeita ao estado uniaxial de tensões. É possível generalizar tal expressão para estados bi ou tridimensionais, mas tal modelo encontra grande aplicação em problemas de conformação mecânica de materiais dúcteis, em que o regime plástico do material é predominante.

Obtém-se o valor da deformação somando-se todas as taxas de variações infinitesimais em comprimento em relação à mudança ocorrida, do início ao fim das deformações. Considerando um estado de tração por simplicidade, tem-se [6]: 
$\mathrm{e}=\frac{\Delta \mathrm{l}_{1}}{\mathrm{l}_{0}}+\frac{\Delta \mathrm{l}_{2}}{\mathrm{l}_{0}+\Delta \mathrm{l}_{1}}+\frac{\Delta \mathrm{l}_{\mathrm{l}}}{\mathrm{l}_{0}+\Delta \mathrm{l}_{1}+\Delta \mathrm{l}_{2}}+\cdots=\sum_{\mathrm{I}_{0}}^{\mathrm{l}_{\mathrm{L}}} \frac{\Delta \mathrm{l}}{\mathrm{l}}$

No limite, o somatório pode ser substituído por uma integração na seguinte forma:

$$
\mathrm{e}=\int_{\mathbb{1}_{\infty}}^{\mathrm{l}_{\mathrm{f}}} \frac{\mathrm{dl}}{\mathrm{l}}=\ln \frac{\mathrm{l}_{\mathrm{f}}}{\mathrm{l}_{0}}
$$

No caso de compressão, como deformações compressivas são definidas como sendo negativas, tem-se:

$$
-\mathrm{e}=\ln \frac{\mathrm{l}_{\mathrm{f}}}{\mathrm{l}_{0}} \text { ou } \epsilon=\ln \frac{\mathrm{l}_{0}}{\mathrm{l}_{\mathrm{f}}}
$$

A medida da deformação verdadeira ou True Strain é bastante versátil e pode-se ignorar o sinal, alocando-se o maior comprimento para o numerador e o menor para o denominador, de forma que a equação assume a forma

$$
\mathrm{e}=\ln \frac{\mathrm{l}_{1}}{\mathrm{l}_{s}}
$$

A deformação verdadeira ajusta-se sobremaneira para grandes deformações em regime plástico, no qual se admite o coeficiente de Poisson igual a 0,5, que resulta na invariância do volume do corpo, ou seja:

$$
V_{0}=A_{0} l_{0}=A_{f} l_{f} \text { ou } \frac{l_{f}}{l_{0}}=\frac{A_{0}}{A_{f}}
$$

Assim,

$$
\mathrm{e}=\ln \frac{\mathrm{l}_{1}}{\mathrm{l}_{g}}=\ln \frac{\mathrm{A}_{0}}{\mathrm{~A}_{\mathrm{f}}}
$$

$\mathrm{Na}$ realidade, a versatilidade da expressão da deformação verdadeira faz com que sua expressão matemática se ajuste para qualquer hipótese sobre o comportamento das deformações secundárias com relação à deformação principal, ou seja, para qualquer valor do coeficiente de Poisson. Além disso, para pequenas deformações mesmo em regime elástico, a deformação verdadeira fornece resultados muito próximos da medida usual de pequenas deformações, conforme será ilustrado posteriormente nos exemplos.

Por fim, a deformação verdadeira é associativa, ou seja, é possível dividir o carregamento e suas respectivas deformações e somá-los que o resultado será equivalente ao da aplicação total da carga [7]. A deformação total é, por definição,

$$
\mathrm{e}_{\mathrm{t}}=\int_{\mathbb{1}_{\mathrm{a}}}^{\mathrm{l}_{\bar{s}}} \frac{\mathrm{dl}}{\mathbb{1}}
$$

Assim, considerando-se a extensão total como soma de uma extensão de $l_{0}$ a $\mathbb{l}_{1}$, e depois de $l_{1}$ a $l_{x}$, a Eq. (31) pode ser expressa como:

$$
e_{\mathrm{t}}=\int_{\mathbb{1}_{a}}^{\mathrm{I}_{1}} \frac{\mathrm{dl}}{\mathrm{I}}+\int_{\mathbb{1}_{1}}^{\mathrm{l}_{\mathrm{s}}} \frac{\mathrm{dl}}{\mathrm{I}}
$$

Caso se faça isso com a medida de pequenas deformações, o resultado será distinto.

\subsection{Relação da True Strain com a Medida de Pequenas Deformações}

É interessante escrever a deformação verdadeira como função da deformação linear. Sabendo-se que:

$$
\mathrm{l}_{\mathrm{f}}=\mathrm{l}_{\mathrm{o}}+\Delta \mathrm{l}
$$

A Eq. (30) pode ser reescrita na forma:

$$
\mathrm{e}=\ln \frac{\mathrm{l}_{0}+\Delta \mathrm{l}}{\mathrm{l}_{0}}=\ln (1+\mathrm{\varepsilon})=\ln \left(1+\frac{\mathrm{du}}{\mathrm{dx}}\right)=\frac{\mathrm{P}}{\mathrm{AE}}
$$

Colocando-se $u(x)$ como função de e:

$$
e^{\left(\frac{\mathbb{P}}{M^{2}}\right)}=1+\frac{d u}{d x} \rightarrow u(x)=x\left(e^{\left(\frac{F}{G E}\right)}-1\right)+A
$$

Note que a primeira derivada de e com relação $x$ é constante, ou seja, mesmo admitindo grandes deformações, o espaçamento entre partículas cresce uniformemente alo longo do corpo, caracterizando o mesmo comportamento de deformação homogênea previsto pela teoria linear:

$$
\mathrm{u}^{\prime}(\mathrm{x})=\left(\mathrm{e}^{\left(\frac{\mathrm{P}}{\mathrm{N}}\right)}-1\right)
$$

No caso de deformação homogênea, sabe-se que na extremidade de uma barra engastada o tensor linear de pequenas deformações fornece:

$$
\varepsilon=\frac{\mathrm{u}_{\max }}{\mathrm{I}_{0}}
$$

Então:

$$
\mathrm{e}=\ln \left(1+\frac{\mathrm{u}_{\max }}{\mathrm{l}_{0}}\right)
$$

\section{Simulações Numéricas}

Considera-se para comparação entre os modelos de deformação uma barra cilíndrica engastada, conforme mostra a Figura 2, com uma carga $P$ aplicada na seção normal da mesma, de comprimento I, área da seção transversal A, módulo de elasticidade E. Este módulo é admitido hipoteticamente constante, mesmo considerando grandes deformações, visando uma melhor comparação dos modelos. 


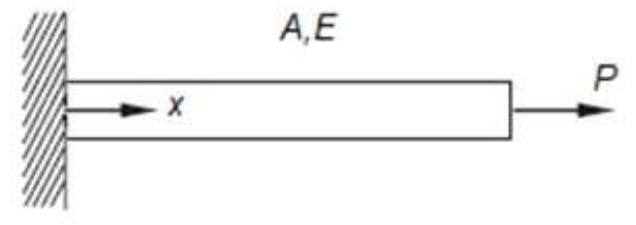

Figura 2- Barra cilíndrica engastada sujeita à carga $P$.

5.1 Exemplo 1 - Obtenção de uma expressão de grandes deformações baseada no tensor de Green para uma barra engastada sob tração.

O tensor de Green $G$ pode ser escrito, para grandes alongamentos e pequenas rotações, como:

$$
G=\varepsilon+\frac{1}{2}(\varepsilon)^{2}=\frac{d u}{d x}+\frac{1}{2}\left(\frac{d u}{d x}\right)^{2}=\frac{P}{A E}
$$

As condições de equilíbrio precisam ser consideradas para gerar a equação de governo. Nesse caso, considerando que o esforço normal é constante, temse:

$$
\frac{d^{2} u}{d x^{2}}+\left(\frac{d u}{d x}\right) \frac{d^{2} u}{d x^{2}}=0
$$

Tomando-se uma função auxiliar $p(x)$ tal que:

$$
\mathrm{p}=\frac{\mathrm{du}}{\mathrm{dx}}
$$

A equação de governo 1.8 assume a forma:

$$
\mathrm{dp}+\mathrm{pdp}=0
$$

Integrando-a, obtém-se:

$$
\mathrm{p}+\frac{\mathrm{p}^{2}}{2}=\mathrm{C}
$$

As raízes dessa última equação 1.12 são:

$$
\mathrm{p}_{1}=(1-\mathrm{C})^{\frac{3}{2}}-1 \text { e } \mathrm{p}_{2}=-(1-\mathrm{C})^{\frac{3}{2}}-1
$$

Ambas as raízes conduzem a mesma solução. Tomando como base p1 e fazendo nova integração chega-se a:

$$
\mathrm{u}=(1-\mathrm{C})^{\frac{2}{2} \mathrm{x}}-\mathrm{x}+\mathrm{D}
$$

Nesta última equação, D é uma nova constante. A primeira condição de contorno inicial impõe que para $\mathrm{x}=0 \rightarrow \mathrm{u}=0$, de que $\mathrm{D}=0$. A outra condição de contorno consiste em:

$$
A E\left[\frac{d u}{d x}+\frac{1}{2}\left(\frac{d u}{d x}\right)^{2}\right]=P
$$

Assim, substituindo a Eq. (45) na Eq. (46), e realizando-se as devidas operações algébricas, obtémse que:

$$
\mathrm{C}=\frac{-2 \mathrm{P}}{\mathrm{EA}}
$$

Finalmente, substituindo-se a relação (47) na Eq. (45):

$$
\mathrm{u}(\mathrm{x})=\left(1+\frac{2 \mathrm{P}}{\mathrm{EA}}\right)^{\frac{2}{2}} \mathrm{x}-\mathrm{x}=\mathrm{x}\left[(1+2 \varepsilon)^{\frac{1}{2}}-1\right]
$$

Percebe-se que devido a sua estrutura matemática, o campo de deslocamentos não cresce uniformemente, ou seja, sua primeira derivada não é constante, não reproduzindo a ideia de deformação homogênea.

5.2 Exemplo 2 - Comparam-se os comportamentos da medida de pequenas deformações, do tensor de Green e da True Strain na barra engastada.

Por clareza, as três medidas são apresentadas a seguir em função da tensão aplicada.

$$
\begin{gathered}
\mathrm{u}(\mathrm{x})=\frac{\mathrm{P}}{\mathrm{AE}} \mathrm{x} \\
\mathrm{u}(\mathrm{x})=\mathrm{x}\left(\mathrm{e}^{\left(\frac{\mathrm{P}}{\mathrm{NE}}\right)}-1\right) \\
\mathrm{u}(\mathrm{x})=\mathrm{x}\left[\left(1+\frac{2 \mathrm{P}}{\mathrm{EA}}\right)^{\frac{1}{2}}-1\right]
\end{gathered}
$$

A Figura 3 mostra a plotagem das três curvas de deformação para diferentes valores de P/AE. Para pequenos valores de deslocamento, as curvas praticamente se equivalem. Todavia, para maiores valores de $\mathrm{P} / \mathrm{AE}$ a deformação verdadeira prevê também maiores deslocamentos, como era de se esperar, pois os denominadores atualizados de cada incremento de deslocamento (vide equação (25)) reduzem a resistência ao esforço. Ao contrário, o tensor de Green enrijece a barra à medida que o esforço se amplia e os deslocamentos aumentam.

Assim, de acordo com a simulação mostrada, a True strain e o tensor de Green resultam em comportamentos distintos e contraditórios. 


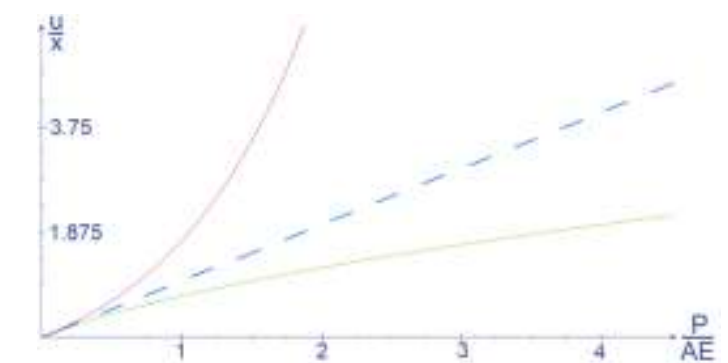

Figura 3- Curvas de deformação nominal linear, dada pela equação $\underline{u}=\mathrm{Ex}$ (em azul), não linear, dada pela Eq. (48) (em verde) e curva de deformação verdadeira (em vermelho), dada pela Eq. (50).

Ressalta-se que nenhuma menção foi feita a atualização da seção reta, que se modifica com a ampliação dos deslocamentos. Assim, em todos os modelos não foi computada a tensão verdadeira ou True Stress, que implicaria tão somente numa correção do valor usado na abscissa do gráfico obtido.

\section{Conclusões}

O desenvolvimento teórico deste trabalho mostrou resumidamente que a deformação verdadeira é um modelo de deformação que possui diversas qualidades. Aproxima-se da teoria linear quando as cargas e deslocamentos resultantes são pequenos; ajusta-se facilmente a comportamentos diferentes da hipótese de conservação de volume, empregados em modelos plásticos - ou seja, opera naturalmente com diferentes valores do coeficiente de Poisson diferentes de 0,5; possui aditividade; representa naturalmente o estado conhecido como deformação homogênea, em que a taxa de espaçamento entre as partículas deformadas numa direção permanece constante; é bastante simples de manipular em estados unidimensionais, mas pode ser ajustada para estados mais complexos, incluindo casos de esforço normal variável. Não é por outra razão que é uma medida consolidada como modelo nos problemas de conformação plástica dos materiais.

Por outro lado, mostrou-se que o tensor de Green no caso de grandes alongamentos resulta matematicamente de uma medida não euclidiana da deformação. Mesmo em casos unidimensionais, não possui consistência conceitual. Pode-se inferir que sua constituição matemática guarda a mesma ideia do tensor de pequenas deformações, apenas ressaltando seu efeito quando os deslocamentos se tornam maiores, por estar elevando o tensor de pequenas deformações ao quadrado. Portanto, não deixa de ser apenas uma forma artificial de amplificar um efeito pequeno, já contabilizado, sem descrever fisicamente qualquer outro fenômeno ligado a alterações significativas na distância relativa entre as partículas do corpo.
Ressalta-se que esta observação se aplica apenas ao exame dos alongamentos, pois nos casos de rotação relevantes no processo de deformação, o tensor de Green denota uma consistência já aferida experimentalmente em diversos problemas da engenharia, como no caso de cordas e cabos.

Tentativas de usar a série de Taylor estendida - com derivada de segunda ordem - foram estudadas, mas não surtiram o efeito desejado, pois a presença da segunda derivada resulta na quebra do conceito de uniformidade dos efeitos de deslocamento numa barra por efeito de uma força constante. Outras inconsistências físicas também se revelaram, particularmente em razão da adoção de três condições de contorno neste caso, que possuem interpretações contraditórias.

Em síntese, o modelo da deformação verdadeira deve ser usado sempre que for possível compatibilizar sua estrutura matemática ao modelo mecânico escolhido. Ressalta-se que no caso de se haverem rotações, a situação é completamente diferente. O tensor de Green capta efeitos rotacionais facilmente observados em estruturas como cordas e cabos e efetivamente verificados em estruturas mais complexas.

\section{Referências}

[1] MALVERN L. E.; Introduction to the Mechanics of a Continuous Medium. New Jersey: Prentice-Hall, 1969.

[2] COIMBRA, A. L.; Lições de Mecânica do Contínuo. São Paulo: Edgar Blucher, 1978.

[3] LIN T. H.; Theory of Inelastic Structures. New York: John Wiley and Sons, 1968.

[4] ODEN, T. J.; Mechanics of Elastic structures. New York: McGraw-Hill, 1967.

[5] TIMOSHENKO, S. P.; GOODIER, J. N.; Theory of Elasticity. New York: McGraw-Hill, 1970.

[6] DATSKO, J.: Properties and Manufacturing Processes. Nova York: John Wiley \& Sons, 1967.

[7] CADDELL, R. M. Deformation and Fracture of Solids. New Jersey: Prentice-Hall,1980.

[8] Why All These Stresses and Strains?, Estocolmo. Disponível em <https://br.comsol.com/blogs/why-allthese-stresses-and-strains>. Acesso em: 03 Jul. 2017. 